\title{
Solid Nodule Appearance as a Predictor of Tumor Spread Through Air Spaces in Patients with Lung Adenocarcinoma: A Propensity Score Matching Study
}

This article was published in the following Dove Press journal:

Cancer Management and Research

Qingpeng Zeng ${ }^{\prime}$
Bingzhi Wang'
Jiagen Li'
Jun Zhao'
Yousheng Mao'
Yushun Gao'
Qi Xue'
Shugeng Gao'
Nan Sun'
Jie He'
'Department of Thoracic Surgery,
National Cancer Center/National Clinical
Research Center for Cancer/Cancer
Hospital, Chinese Academy of Medical
Sciences and Peking Union Medical
College, Beijing, People's Republic of
China; ${ }^{2}$ Department of Pathology,
National Cancer Center/National Clinical
Research Center for Cancer/Cancer
Hospital, Chinese Academy of Medical
Sciences and Peking Union Medical
College, Beijing, People's Republic of
China

Correspondence: Jie He

Department of Thoracic Surgery, National Cancer Center/National Clinical Research Center for Cancer/Cancer Hospital,

Chinese Academy of Medical Sciences and

Peking Union Medical College, No. 17,

Panjiayuan Nanli, Beijing I0002I, People's

Republic of China

Email prof.jiehe@gmail.com
Objective: Spread through air spaces (STAS) has been reported to be an invasive histological pattern with poor prognosis in lung cancer; however, little is known about its intrinsic risk factors. This work analyzed the correlation between pathological and radiological features and STAS in resected lung adenocarcinomas.

Patients and Methods: We retrospectively reviewed 1821 consecutive surgically treated patients with histologically diagnosed lung adenocarcinoma (174 positive for STAS and 1647 negative for STAS) from December 2017 to November 2018 at our institution. Propensity score matching identified 170 well-balanced pairs of patients. The correlations between pathological and radiological features and the presence of STAS were analyzed.

Results: Before propensity matching, the incidence rate of STAS was $9.6 \%$ in all patients. In matched cohorts, multivariate analysis showed that the presence of STAS was significantly correlated with pure solid nodules $(\mathrm{SNs})(p=0.001)$ and solid/micropapillary patterns (SMPs) $(p=0.002)$. The odds ratio for STAS in SN-positive and SMP-positive adenocarcinoma against that in SN-negative and SMP-negative adenocarcinoma was 10.922 (95\% confidence interval, 5.826-20.475; $p<0.001)$. Tumor differentiation, visceral pleural invasion (VPI), lymphovascular invasion (LVI), invasive adenocarcinoma, and non-lepidic subtype were significantly associated with STAS in the univariate analysis $(p<0.05)$; however, the differences failed to reach a significant level in the multivariate analysis.

Conclusion: We found that STAS was significantly correlated with several invasive clinicopathological patterns. The presence of SNs and SMPs were revealed as independent predictors for STAS, which could offer clinicians clues to identify STAS-positive adenocarcinoma.

Keywords: lung adenocarcinoma, spread through air spaces, solid pulmonary nodule

\section{Introduction}

The non-small cell lung cancer (NSCLC) still ranks the first common and the leading cause of cancer-related deaths word widely. ${ }^{1}$ As the major subtype of NSCLC, lung adenocarcinoma (ADC) presents with diverse histological patterns and molecular features. In recent decades, based on morphology, immunohistology, and molecular analyses, several clinicopathological features have been proven to be correlated with the prognosis of lung ADC, which is of vital significance in clinical therapeutic decision making. ${ }^{2}$ Tumor spread through air spaces (STAS), which is 
described as the spread of cancer cells into air spaces in the lung parenchyma beyond the edge of the main tumor, was first introduced by the 2015 WHO classification of lung tumors and further validated and analyzed. ${ }^{3}$ Several studies have reported the prognostic value of STAS and revealed its correlation with certain clinicopathological characteristics; thus, STAS was gradually recognized as a novel invasive pattern of ADC. ${ }^{4-6}$

However, the existence of STAS remains controversial, as some researchers consider STAS to be an artifact of tissue processing rather than an indicator of certain pathological change. ${ }^{7}$ Further comprehensive studies and accurate definitions are needed to settle this dispute concerning STAS. In addition, little is known about the radiological features and genetic mutations associated with STAS in lung ADC. Thus, we analyzed the clinicopathological features of surgically resected lung ADC with STAS to explore these intrinsic correlations.

\section{Patients and Methods}

\section{Patient Cohort}

We reviewed a large, single-institution database of patients with lung cancer in the Cancer Institute and Hospital of the Chinese Academy of Medical Sciences from December 2017 to November 2018. Our present study included patients who underwent surgical resection for lung cancer and histologically confirmed primary lung ADC. A total of 1841 consecutive patients were identified based on this selection. We excluded cases lacking complete medical records $(\mathrm{n}=3)$, received preoperative neoadjuvant therapy $(\mathrm{n}=12)$, accompanying with other malignancies or histological components $(\mathrm{n}=5)$. After the exclusion, 1821 patients met our criteria and enrolled in the study cohort. The criteria for patient selection and exclusion were also shown in Supplementary Figure S1. Patients' demographics, pathological/radiological features, and genetic parameters were retrieved from the medical record database and analyzed.

\section{Radiological Features}

A thin-section chest computed tomography (CT) scan was conducted in all patients within one month preoperatively. Examinations were performed using 64-detector row scanners (LightSpeed VCT, Discovery CT750 HD or Optima CT660, General Electric Medical Systems; TOSHIBA Aquilion, TOSHIBA Medical Systems) at full inspiration. The CT parameters were as follows: tube voltage, 120
$\mathrm{kVp}$; auto mA settings (tube current, $200-350 \mathrm{~mA}$, noise index, 13; pitch, 0.992 or 0.984 ; rotation time, $0.5 \mathrm{~s}$; thickness, $5 \mathrm{~mm}$ ). The image was reconstructed with $1.25 \mathrm{~mm}$-thick slices using a standard reconstruction algorithm. The pulmonary nodule was subclassified into three categories, namely, pure ground-glass nodules (GGNs), part-solid nodules (PSNs), and pure solid nodules (SNs), according to the proportion of the solid component. PSNs were defined as lesions with both ground-glass opacity (GGO) and a solid component. ${ }^{8}$ The nodule features of all enrolled cases were independently reviewed by a thoracic radiologist (Dr. Yang) and a thoracic surgeon (Dr. Zeng). The two reviewers were both blinded to the STAS status before analysis, and the final assessment was made through a discussion if there was a disagreement.

\section{Histopathologic Examination}

The histopathologic classification of lung ADC was according to 2015 WHO criteria, including adenocarcinoma in situ (AIS), minimally invasive adenocarcinoma (MIA), and invasive adenocarcinoma which was further classified into five subtypes (lepidic, acinar, papillary, solid, and micropapillary). The percentage of each subtype was recorded in a semiquantitative manner (in 5\% increments), and the predominant pattern refers to the major component with the largest percentage. Tumors without a solid or micropapillary pattern (SMP) were defined as SMP negative, tumors containing an SMP $>5 \%$ but did not have an SMP as the predominant pattern were defined as SMP minor, and those with SMP as the predominant subtype were classified as SMP predominant. The definitions of other features including tumor differentiation, visceral pleural invasion (VPI), and lymphovascular invasion (LVI) were described in the previous report. ${ }^{9}$ TNM stage was based on the American Joint Committee on Cancer (AJCC) 8th edition staging manual. ${ }^{10}$ STAS was defined as detached tumor cells within alveolar spaces that were separated from the main tumor. The pattern of STAS may be presented in the morphologic forms of single cells, micropapillary clusters, or solid nests, according to Kadota's criteria. ${ }^{4}$ Epidermal growth factor receptor (EGFR) mutation and kirsten rat sarcoma (KRAS) mutation were analyzed through direct DNA sequencing or Mutation Detection Kit in selected cases depending on clinical needs. We reviewed these data from each patient's postoperative pathological report, which was conducted by at least two board-certified pathologists. Besides, the hematoxylin-eosin (HE) staining sections of all enrolled cases were reviewed by another independent pathologist (Dr. Wang), 
who was blind to the pathological reports. The disagreements were resolved by consensus of Dr. Wang with the initial reviewers.

\section{Propensity Score Matching}

On the basis of the imbalanced sample size and potential selection bias in this nonrandomized cohort, we adopted the propensity score matching to select comparable cases from the STAS-negative group for further analysis. Propensity scores were computed as the conditional probability of the presence of STAS using a multivariable logistic regression model. Age at diagnosis, sex, smoking status, forced vital capacity (FVC)/predicted, forced expiratory volume in 1 second (FEV1)/predicted, carcinoembryonic antigen (CEA), and pathologic tumor size were included as covariates to balance the two groups. The balance in covariates was assessed by the absolute standardized mean difference (SMD) before and after matching. An SMD $\leq 0.1$ indicates that the covariate between the two matched groups was well balanced. ${ }^{11}$ We performed $1: 1,1: 2$, and $1: 3$ ratio matching, and $1: 1$ ratio matching was chosen because it achieved the most balanced data set. Propensity score matching was performed with PSMATCHING (add-on for SPSS, version 3.04) and underlying $\mathrm{R}$ packages (version 3.1.0).

\section{Statistical Analysis}

Continuous variables were expressed as mean \pm standard deviation (SD) or median \pm range and compared using Student's $t$-test or nonparametric Mann-Whitney $U$-test according to the normality of data. Categoric variables were presented as frequency and percentage and compared using the Chi-square test or Fisher's exact test. A multivariate logistic regression analysis was performed to identify independent predictive factors of STAS among the correlated variables from the univariate analysis. Statistical analysis was performed using SPSS (IBM, Version 23.0, Armonk, NY, USA). A p-value $<0.05$ was considered statistically significant.

\section{Results}

\section{Propensity Score Matching results and Patient Characteristics}

From December 2017 to November 2018, a total of 1821 patients (174 cases in the STAS-positive group and 1647 cases in the STAS-negative group) were enrolled into analysis, and the incidence rate of STAS was $9.6 \%$.
Before propensity score matching, the baseline variables between the two groups were unbalanced, with a mean propensity score of 0.090 for the STAS-negative group and 0.145 for the STAS-positive group (SMD =0.554). After 1:1 propensity matching using the nearest neighbor matching algorithm (caliper $=0.05), 170$ matched pairs $(340$ cases) were finally included in the analysis. All included covariates were well balanced (SMD $<0.01)$ with an equal mean propensity score of 0.137 (Supplementary Table S1). For a more intuitive presentation, we also show the distribution of the propensity scores and SMDs for both groups before and after propensity matching in Supplementary Figure S2.

The clinical characteristics of the patients in the two groups are shown in Supplementary Table S1. The mean age of all matched patients was 59.0 years (range, 31-82 years), including 148 men (43.5\%) and 192 women (56.5\%). Patients who never smoked (234 of 340, $68.8 \%$ ) were predominant in our study. No significant differences were found between the two groups in lung cancer family history and other comorbidities before or after matching. A minority of patients (96 of 340, 28.2\%) experienced abnormally elevated CEA levels and achieved balanced covariates. To achieve a better comparison of the pathological or radiological features between patients with or without STAS, tumor size was also included in the covariates. After matching, the two groups had similar tumor sizes, with a median of $2.1 \mathrm{~cm}$ (range, $0.5-8.5 \mathrm{~cm}$ ).

\section{Pathological and Radiological Features According to STAS Status}

The pathological and radiological features according to STAS status are summarized in Table 1, and examples of three cases of lung ADC with STAS stratified by different CT appearances are shown in Figure 1. In terms of pathological characteristics, STAS was found to be significantly associated with tumor differentiation, VPI, and LVI $(p<$ 0.05). Lymph nodal metastasis was observed more frequently in STAS $(+)$ patients $(29.4 \%, 50$ of 170$)$ than in STAS (-) patients $(20.6 \%, 35$ of 170$)$; however, the difference between the two groups failed to reach significant level ( $p=0.079)$. Moreover, the two groups did not differ in pathological TNM stage $(p=0.211)$. Similar rates of multiple primary lung lesions were found in STAS (-) and STAS $(+)$ patients [8.8\% (15 of 170$)$ vs $10.6 \%$ (18 of 170$)$, $p=0.715]$. Notably, no AIS or MIA was found in STAS $(+)$ patients, which was in obvious contrast to STAS (-) 
Table I Comparison of Pathological Characteristics, Radiological Features, and Genetic Mutations Between STAS (-) and STAS (+) Groups After Propensity Matching

\begin{tabular}{|c|c|c|c|c|}
\hline Characteristics & Matched Patients & STAS (-) & STAS (+) & $P$ value \\
\hline No. of patients & 340 & 170 & 170 & \\
\hline Tumor differentiation & & & & $<0.001^{\mathrm{a}}$ \\
\hline Well & 26 & $24(14 . I)$ & $2(1.2)$ & \\
\hline Moderate/poor & 288 & $126(74.1)$ & $162(95.3)$ & \\
\hline $\mathrm{N} / \mathrm{A}$ & 26 & $20(11.8)$ & $6(3.5)$ & \\
\hline Bronchial involvement & & & & 0.496 \\
\hline Positive & 39 & $17(10.0)$ & $22(12.9)$ & \\
\hline Negative & 301 & $153(90.0)$ & I $48(87.1)$ & \\
\hline VPI & & & & 0.005 \\
\hline Positive & 94 & $35(20.6)$ & $59(34.7)$ & \\
\hline Negative & 246 & I35 (79.4) & III (65.3) & \\
\hline LVI & & & & 0.029 \\
\hline Positive & 67 & $25(14.7)$ & $42(24.7)$ & \\
\hline Negative & 273 & $145(85.3)$ & $128(75.3)$ & \\
\hline Lymph nodal metastasis & & & & 0.079 \\
\hline Yes & 85 & $35(20.6)$ & $50(29.4)$ & \\
\hline No & 255 & $135(79.4)$ & $120(70.6)$ & \\
\hline Pathological stage & & & & $0.211^{\mathrm{a}}$ \\
\hline AIS & 1 & $\mathrm{I}(0.6)$ & 0 & \\
\hline 1 & 228 & $|2|(7 \mid .2)$ & $107(62.9)$ & \\
\hline II & 45 & $22(12.9)$ & $23(13.5)$ & \\
\hline III & 56 & $21(12.4)$ & $35(20.6)$ & \\
\hline IV & 10 & $5(2.9)$ & $5(2.9)$ & \\
\hline Multiple primary lesion & & & & 0.715 \\
\hline Yes & 33 & $15(8.8)$ & $18(10.6)$ & \\
\hline No & 307 & 155 (9I.2) & $152(89.4)$ & \\
\hline Adenocarcinoma subtypes & & & & $0.010^{\mathrm{a}}$ \\
\hline AIS & 1 & $\mathrm{I}(0.6)$ & 0 & \\
\hline MIA & 8 & $8(4.7)$ & 0 & \\
\hline Invasive adenocarcinoma & 321 & $156(9 \mid .8)$ & $165(97.1)$ & \\
\hline Variants of invasive adenocarcinoma & 10 & $5(2.9)$ & $5(2.9)$ & \\
\hline Predominant subtype & & & & $<0.001$ \\
\hline Lepidic predominant & 22 & $18(10.6)$ & $4(2.4)$ & \\
\hline Acinar predominant & 176 & $93(54.7)$ & $83(48.8)$ & \\
\hline Papillary predominant & 78 & $31(18.2)$ & $47(27.6)$ & \\
\hline Solid predominant & 29 & $10(5.9)$ & $19(11.2)$ & \\
\hline Micropapillary predominant & 11 & 0 & II (6.5) & \\
\hline$N / A$ & 24 & $18(10.6)$ & $6(1.8)$ & \\
\hline Solid/micropapillary pattern & & & & $<0.00 \mathrm{I}^{\mathrm{a}}$ \\
\hline SMP negative & 134 & $96(56.5)$ & $38(22.4)$ & \\
\hline SMP predominant & 40 & $10(5.9)$ & $30(17.6)$ & \\
\hline SMP minor & 157 & $56(32.9)$ & $101(59.4)$ & \\
\hline N/A & 9 & $8(4.7)$ & I (0.6) & \\
\hline
\end{tabular}

(Continued) 
Table I (Continued).

\begin{tabular}{|c|c|c|c|c|}
\hline Characteristics & Matched Patients & STAS (-) & STAS (+) & $P$ value \\
\hline EGFR mutation & & & & 0.486 \\
\hline Positive & 195 & $101(59.4)$ & $94(55.3)$ & \\
\hline Wild type & 120 & $55(32.4)$ & $65(38.2)$ & \\
\hline N/A & 25 & $14(8.2)$ & II (6.5) & \\
\hline EGFR mutation type & & & & 0.201 \\
\hline Wild type & 120 & $55(32.4)$ & $65(38.2)$ & \\
\hline Exon 19 deletion & 90 & $54(31.8)$ & $36(21.2)$ & \\
\hline Exon 21 missense & 91 & $4 \mid(24.1)$ & $50(29.4)$ & \\
\hline Miscellaneous & 14 & $6(3.5)$ & $8(4.7)$ & \\
\hline KRAS mutation & & & & 0.347 \\
\hline Positive & 35 & $21(12.4)$ & $14(8.2)$ & \\
\hline Wild type & 280 & I35 (79.4) & $145(85.3)$ & \\
\hline N/A & 25 & $14(8.2)$ & II (6.5) & \\
\hline Nodule pattern & & & & $<0.00 \mathrm{I}^{\mathrm{a}}$ \\
\hline Pure GGN & 27 & $26(15.3)$ & $\mathrm{I}(0.6)$ & \\
\hline PSN & 99 & $69(40.6)$ & $30(17.6)$ & \\
\hline SN & 214 & $75(44.1)$ & $139(81.8)$ & \\
\hline
\end{tabular}

Note: a Comparison between variables was performed using the Fisher exact test.

Abbreviations: STAS, spread through air spaces; VPI, visceral pleural invasion; LVI, lymphovascular invasion; AIS, adenocarcinoma in suit; MIA, minimally invasive adenocarcinoma; SMP, solid/micropapillary pattern; GGN, ground glass nodule; PSN, part-solid nodule; SN, solid nodule; N/A, non-applicable.

patients $(5.3 \%, 9$ of $170, p=0.010)$. Furthermore, in terms and micropapillary predominant tumors $(6.5 \%, 11$ of 170 , of histological subtypes, STAS was more frequently found $p<0.001)$ but was rarely found in lepidic predominant in solid predominant tumors $(11.2 \%, 19$ of $170, p=0.080) \quad$ tumors $(2.4 \%, 4$ of $170, p=0.002)$. We also performed
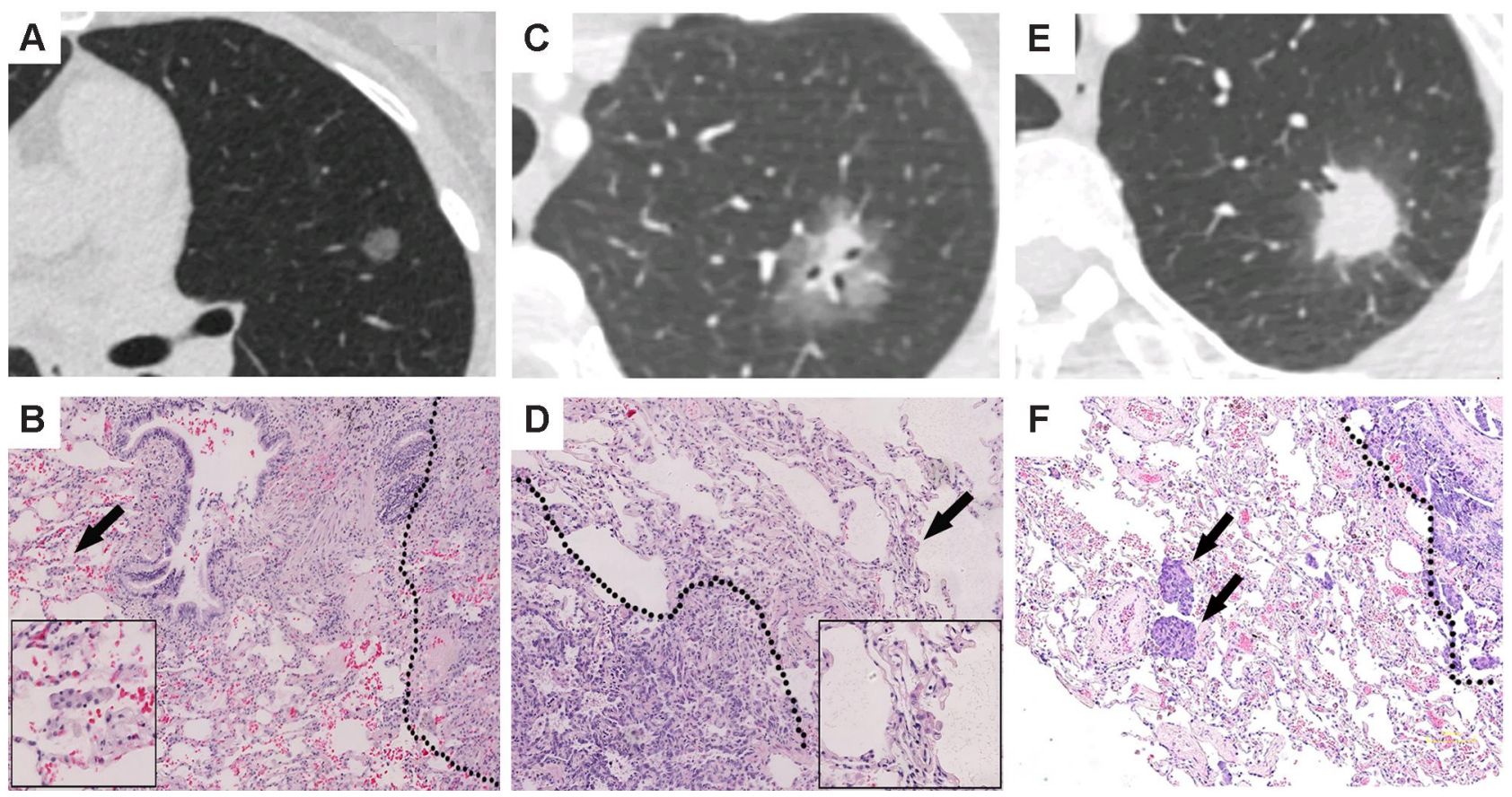

Figure I CT features (width, $1600 \mathrm{HU}$; level, $-600 \mathrm{HU}$ ) and their corresponding STAS manifestations: (A) Pure GGN; (C) PSN; and (E) SN. (B, D and F) show the corresponding STAS features of the above nodules, respectively. Photomicrograph shows detached clusters of tumor cells (arrows) detached within alveolar spaces beyond the edge of the main tumor (dotted lines) by hematoxylin-eosin staining (magnification, $\times 100$ ). Boxed region in (B and $\mathbf{D})$ shows the high-power view of the STAS feature (magnification, $\times 400$ ).

Abbreviations: CT, computed tomography; HU, Hounsfield unit; STAS, spread through air spaces; GGN, ground-glass nodule; PSN, part-solid nodule; SN, solid nodule. 
Table 2 Multivariate Analysis of the Correlation of Pathological Characteristics, Radiological Features and Genetic Mutations with the Presence of STAS

\begin{tabular}{|c|c|c|c|c|c|}
\hline & No. & STAS (+) & OR & $95 \% \mathrm{Cl}$ & $P$ value \\
\hline $\begin{array}{l}\text { Tumor differentiation } \\
\text { Well } \\
\text { Moderate/poor }\end{array}$ & $\begin{array}{l}26 \\
288\end{array}$ & $\begin{array}{l}2(1.2) \\
162(95.3)\end{array}$ & $\begin{array}{l}1.000 \\
5.451\end{array}$ & $0.915-32.484$ & 0.063 \\
\hline $\begin{array}{l}\text { VPI } \\
\text { Positive } \\
\text { Negative }\end{array}$ & $\begin{array}{l}94 \\
246\end{array}$ & $\begin{array}{l}59(34.7) \\
111(65.3)\end{array}$ & $\begin{array}{l}1.000 \\
0.750\end{array}$ & $0.432-1.301$ & 0.306 \\
\hline $\begin{array}{l}\text { LVI } \\
\text { Positive } \\
\text { Negative }\end{array}$ & $\begin{array}{l}67 \\
273\end{array}$ & $\begin{array}{l}42(24.7) \\
128(75.3)\end{array}$ & $\begin{array}{l}1.000 \\
1.200\end{array}$ & $0.643-2.237$ & 0.567 \\
\hline $\begin{array}{l}\text { Adenocarcinoma subtypes } \\
\text { Invasive adenocarcinoma } \\
\text { Others }\end{array}$ & $\begin{array}{l}321 \\
19\end{array}$ & $\begin{array}{l}165(97.1) \\
5(2.9)\end{array}$ & $\begin{array}{l}1.000 \\
0.663\end{array}$ & $0.056-7.817$ & 0.744 \\
\hline $\begin{array}{l}\text { Predominant subtype } \\
\text { Lepidic } \\
\text { Non-lepidic }\end{array}$ & $\begin{array}{l}22 \\
294\end{array}$ & $\begin{array}{l}4(2.4) \\
160(97.6)\end{array}$ & $\begin{array}{l}1.000 \\
0.565\end{array}$ & $0.129-2.479$ & 0.449 \\
\hline $\begin{array}{l}\text { Solid/micropapillary pattern } \\
\text { SMP negative } \\
\text { SMP positive }\end{array}$ & $\begin{array}{l}134 \\
197\end{array}$ & $\begin{array}{l}38(22.4) \\
131 \text { (77.I) }\end{array}$ & $\begin{array}{l}1.000 \\
2.575\end{array}$ & $1.412-4.696$ & 0.002 \\
\hline $\begin{array}{l}\text { Patterns of nodule } \\
\text { Pure GGN or PSN } \\
\text { SN }\end{array}$ & $\begin{array}{l}126 \\
214\end{array}$ & $\begin{array}{l}31(18.2) \\
139(81.8)\end{array}$ & $\begin{array}{l}1.000 \\
3.223\end{array}$ & $|.803-5.76|$ & $<0.001$ \\
\hline
\end{tabular}

Abbreviations: STAS, spread through air spaces; OR, odds ratio; Cl, confidence interval; SMP, solid/micropapillary pattern; GGN, ground-glass nodule; PSN, partsolid nodule; SN, solid nodule.

a subanalysis of SMPs between the two groups and found that $131(77.1 \%)$ STAS (+) patients showed predominant or minor SMP, whereas only 38 (22.4\%) STAS $(+)$ patients were negative for SMPs $(p<0.001)$. Information on genetic mutations was available in 315 (92.6\%) patients, and no significant difference was observed in either EGFR
A

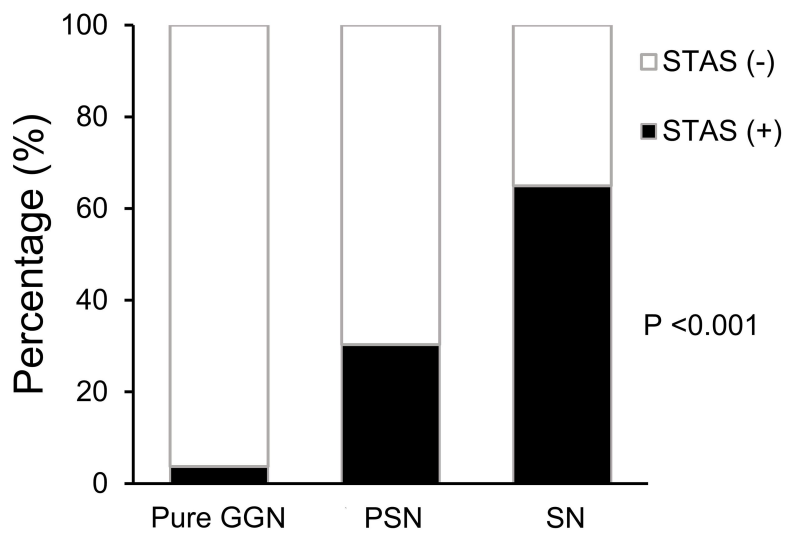

B

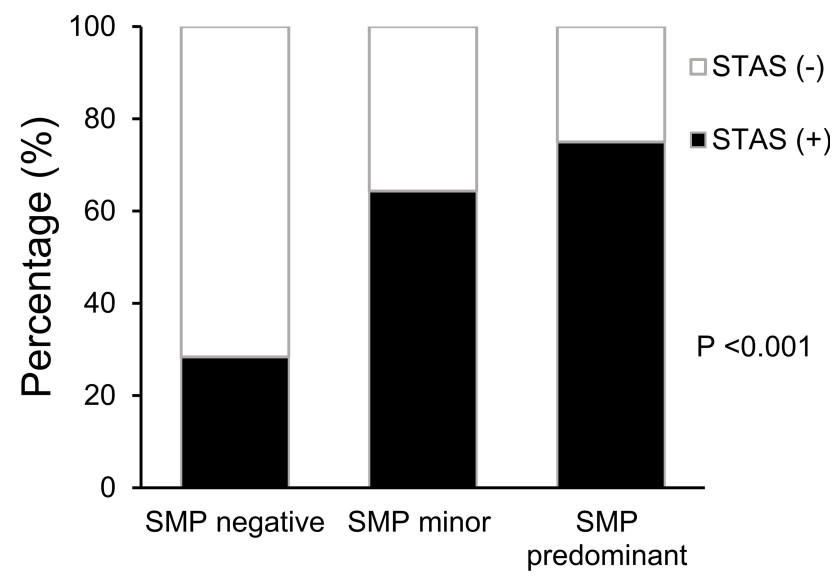

Figure 2 The histogram shows the relative proportions of STAS stratified by increases in solid components (A) or SMPs (B). The frequency of STAS increased in proportion with the solid components and SMPs $(p<0.001)$.

Abbreviations: STAS, spread through air spaces; GGN, ground-glass nodule; PSN, part-solid nodule; SN, solid nodule; SMP, solid/micropapillary pattern. 
Table 3 ORs for STAS According to the Presence or Absence of SNs with SMPs

\begin{tabular}{|l|l|l|l|l|l|}
\hline & No. & STAS (+) & OR & $\mathbf{9 5 \%}$ Cl & P value \\
\hline SN (-)/SMP (-) & 27 & $1(0.6)$ & 1 & & \\
SN (+)/SMP (-) or SN (-)/SMP (+) & 107 & $37(21.8)$ & 3.495 & $1.751-6.976$ & $<0.001$ \\
SN (+)/SMP (+) & 197 & $131(77.1)$ & 10.922 & $5.826-20.475$ & $<0.001$ \\
\hline
\end{tabular}

Abbreviations: STAS, spread through air spaces; SN, pure solid nodule; SMP, solid/micropapillary pattern; OR, odds ratio; Cl, confidence interval.

mutations or KRAS mutations between STAS (-) and STAS $(+)$ patients ( $p=0.486, p=0.347$, respectively). When the EGFR mutations were further subdivided into wild type, exon 19 deletion, exon 21 missense, or miscellaneous, no significant differences were observed $(p=$ $0.210)$. In terms of radiological features, the distribution of nodule patterns differed between the two groups $(p<$ $0.001)$. Only $1(0.6 \%)$ STAS $(+)$ patient showed a pure GGN, whereas nearly all STAS $(+)$ tumors manifested as either SNs $(81.8 \%, 139$ of 170$)$ or PSNs $(17.6 \%, 30$ of $170)$. Correspondingly, a relatively lower rate $(44.1 \%, 75$ of 170) of SN patterns was found in STAS (-) tumors.

\section{Multivariate Analysis of the Correlation Between Pathological and Radiological Features and the Presence of STAS}

The multivariate logistic regression model included variables that were significant in the univariate analysis: tumor differentiation, VPI, LVI, adenocarcinoma subtype, lepidic predominant subtype, SMP, and nodule pattern. STAS was significantly correlated with SMPs and nodule patterns in the multivariate analysis $(p<0.01)$. As shown in Table 2, the presence of STAS showed a significant correlation with SMP-positive tumors (odds ratio [OR], 2.575; 95\% confidence interval [CI], 1.412-4.696; $p=0.002)$ and SNs (OR, 3.223; 95\% CI, 1.803-5.761; $p<0.001$ ). However, the other included parameters failed to reach significance. When stratifying the tumors according to the SMP or nodule pattern, the frequency of STAS increased as the frequency of SMPs or solid appearances on imaging increased, both $p<0.001$ (Figure 2).

\section{ORs for STAS Based on the Presence or Absence of SNs and SMPs}

Additionally, we also analyzed the ORs for STAS based on the presence or absence of SNs and SMPs. In comparison with SN (-)/SMP (-) adenocarcinomas, the OR for STAS in $\mathrm{SN}(+) / \mathrm{SMP}(-)$ or SN (-)/SMP $(+)$ adenocarcinomas was $3.495(95 \% \mathrm{CI}, 1.751-6.976, p<0.001)$, and the OR for STAS in $\mathrm{SN}(+) / \mathrm{SMP}(+)$ adenocarcinomas was 10.922 (95\% CI, 5.826-20.475, $p<0.001$ ), as shown in Table 3 and Figure 3.

\section{Discussion}

In the current study, STAS was observed in 174 of 1821 (9.6\%) cases of surgically resected lung ADC and was revealed to be significantly associated with certain histological characteristics, especially SMPs. In addition, a solid appearance on CT was also revealed as an independent predictive parameter for STAS in tumors. Our findings show that STAS-positive ADC suggests aggressiveness and warrants intensive management; moreover, this tumor type may be predicted with the combination of an SN appearance with a pathological SMP.

The pathological phenomenon of STAS was recently recognized and then extensively studied. Among all the published studies, the definition of STAS has slightly changed. Onozato et $\mathrm{al}^{12}$ implied tumor nests to be the

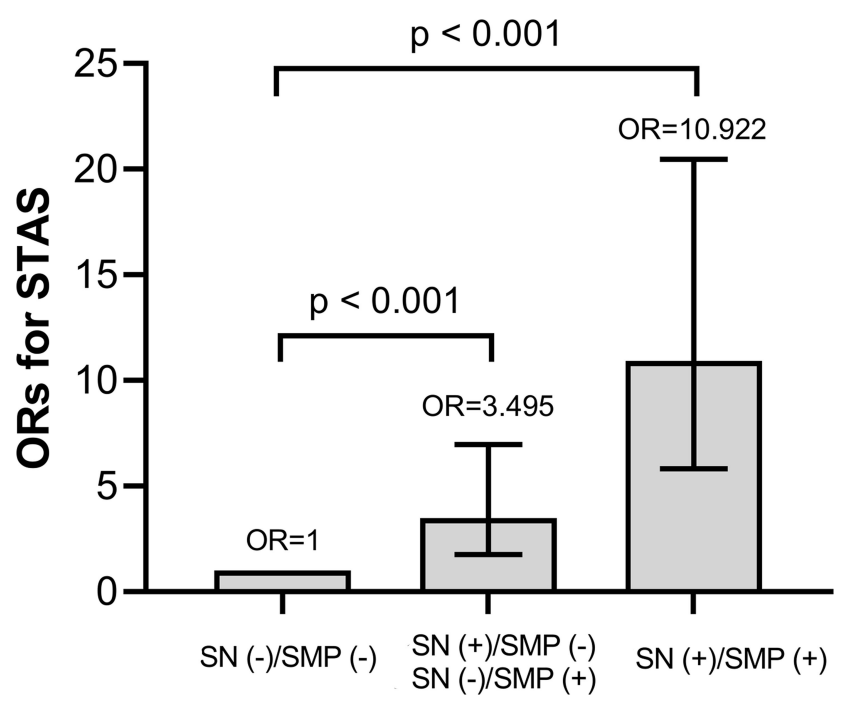

Figure 3 According to the presence or absence of SNs and SMPs, the following three groups were created: the SN (-)/SMP (-) group, the SN (+)/SMP (-) or SN $(-) / S M P(+)$ group, and the SN (+)/SMP (+) group. The ORs for STAS are shown as histograms using the SN (-)/SMP (-) group as a reference.

Abbreviations: SN, solid nodule; SMP, solid/micropapillary pattern; STAS, spread through air spaces; OR, odds ratio. 
criterion for STAS. Kadota et $\mathrm{al}^{4}$ subsequently broadened the definition to include cell clusters or even single cells, which were henceforth widely applied. Several studies have revealed the negative effect of STAS on prognosis. The initial two large cohorts ${ }^{2,4}$ concerning STAS in lung ADC both indicated that STAS was significantly associated with reduced overall survival. Then, several subsequent studies also confirmed the negative impact of STAS on patient survival as well as on cancer recurrence in early-stage lung ADC. ${ }^{13-17}$ Thus, as a certain invasive pattern, the significance of STAS was further acknowledged.

STAS has also been studied for its correlation with clinicopathological factors in lung ADC (Supplementary Table $\underline{\mathrm{S}}$ ). In terms of clinical characteristics, the results showed obvious heterogeneity among previous studies, as only a few reported STAS to be associated with the male sex, smoking, or elevated CEA levels. ${ }^{2,13,14}$ Hence, we first applied a propensity score matching method to balance the baseline features of enrolled patients to achieve better analysis results. Although not significant in the multivariate logistic analysis, STAS was correlated with tumor differentiation, VPI, LVI, the invasive adenocarcinoma subtype, and papillary or micropapillary predominant $\mathrm{ADC}$ in the univariate analysis after matching. As noninvasive subtypes, AIS and MIA have been confirmed to indicate a favorable prognosis with a 5 -year survival rate near $100 \%{ }^{18}$ In our study, no STAS was found in cases of either AIS or MIA, which seems reasonable for noninvasive histological patterns. However, Toyokawa et $\mathrm{al}^{17}$ reported that the incidence of atypical adenomatous hyperplasia (AAH)/AIS/ MIA subtypes in STAS-positive patients was 5.8\% (11 of 191). Therefore, it appears premature to confirm that STAS is absent in noninvasive ADC subtypes. As a more aggressive histological pattern of invasive adenocarcinoma, the SMP was proven to indicate an unfavorable prognosis, even if it was not the predominant subtype. ${ }^{19,20}$ Therefore, we also investigated the difference in SMPs between the STAS (-) and STAS (+) groups. In the final multivariate analysis, the SMP was the only pathological parameter associated with STAS. The proportion of STAS (+) SMP-positive patients was significantly higher than that of STAS (+) SMP-negative patients $(77.1 \%$ vs $22.4 \%$; OR, 2.575; $p=0.002$ ). These results are inconsistent with other previous studies concerning STAS in lung ADC. ${ }^{2,4,6,9,13-17,21,22}$ It is noteworthy that several studies also suggested that STAS was associated with a larger tumor size. Uruga et $\mathrm{al}^{15}$ revealed that in 208 cases of stage I (tumor size $\leq$ $2 \mathrm{~cm}$ ) lung ADC, STAS was significantly correlated with a larger tumor size $(p=0.037)$. Toyokawa et $\mathrm{al}^{16}$ also revealed that STAS positivity was correlated with a pathologically larger tumor size $(p=0.004)$. Regretfully, both studies were based on cohorts containing early-stage lung ADC only. Warth et $\mathrm{al}^{2}$ reported that in 569 cases of stage I to IV lung ADC, the presence of STAS was associated with a higher $\mathrm{N}$ and $\mathrm{M}$ stage but not $\mathrm{T}$ stage. Kim et $\mathrm{al}^{21}$ reached similar results in a cohort of 316 stage I to III cases. The contradictions among those studies may be attributed to the heterogeneity of the enrolled patients, and perhaps nodal metastasis may influence the correlation between STAS and TNM stage. Hu et $\mathrm{al}^{9}$ reported that in a multivariate analysis of 500 cases of lung ADC, STAS was significantly correlated with $\mathrm{T}$ stage $(p=$ $0.001)$ and $\mathrm{N}$ stage $(p=0.032)$. To further study the underlying pathological factors associated with STAS, we enrolled all pathological stages from AIS to stage IV tumors, and the tumor size was also balanced with propensity matching. Although significant differences existed before propensity matching, similar distributions were found for both lymph node metastasis $(p=0.079)$ and TNM stage $(p=0.211)$ between the STAS (+) and STAS (-) groups after matching. Thus, the relationships between STAS and nodal metastasis and TNM stage remain controversial and warrant further study in large cohorts.

The association between STAS and genetic features in lung $\mathrm{ADC}$ remains unclear due to the limited number of relevant studies and lack of adequate data. Previous studies revealed that STAS had a significant relationship with wild-type EGFR. $^{2,6,12,13,21}$ In addition, two of these studies also stated that KRAS and BRAF mutations were found predominantly in STAS-positive patients. ${ }^{2}$ Although not routinely tested for, lung ADC-related driver mutations were analyzed in most surgically resected cases in our institution. We found that STAS was prone to exist in wild-type EGFR or wild-type KRAS tumors; however, the difference between the two groups failed to reach a significant level. Toyokawa et $\mathrm{al}^{17}$ also reported that the presence of STAS showed no significant association with EGFR mutations or PD-1 expression. To date, no previous studies have analyzed the detailed EGFR mutation types, so we conducted a subanalysis of different EGFR mutation types (including wild type, exon 19 deletion, exon 21 missense, and others); however, no differences were found between the two groups.

Several studies have confirmed the adverse effect of STAS on postoperative recurrence in lung ADCs treated with limited resection. ${ }^{4,5,16,23,24}$ In case of that, limited resection for STASpositive ADCs should be cautiously considered. The detection of STAS preoperatively seems vitally important for clinicians to achieve optimal therapeutic planning. Intraoperative frozen 
section pathology appears to be a feasible method; however, its feasibility remains unclear. In addition to the main tumor, the non-neoplastic lung parenchyma is also required in the frozen section specimen of STAS analysis. In 2017, Kameda et $\mathrm{al}^{25}$ conducted a study evaluating STAS with frozen sections and reported satisfactory sensitivity, specificity, and accuracy $\left(71 \%, 92.4 \%\right.$, and $80 \%$, respectively). However, Walts et $\mathrm{al}^{26}$ reported a low sensitivity of $50 \%$ and a high specificity of $100 \%$. In a recent propensity score-matched analysis of $698 \mathrm{~T} 1$ lung ADCs, Eguchi et $\mathrm{al}^{27}$ selected cases with adequate normal lung parenchyma in frozen sections to detect STAS and reported a relatively better sensitivity $(71 \%)$ and specificity (92\%). These findings preliminarily indicate that the detection of STAS through frozen section analysis is feasible and should be further validated in prospective studies given the sparsely available data.

In addition to intraoperative frozen section analysis, preoperative radiological features have also been revealed to be a potential predictor for STAS. Several studies have indicated that the solid appearance of a nodule on CT represents a more invasive pattern and worse prognosis than a GGN appearance. $^{28,29}$ Some investigators also suggested that different percentages of solid components (calculated by the consolidation to tumor ratio, CTR) represent distinct prognoses in part-solid lung ADCs. ${ }^{30,31}$ However, in recent published studies conducted by Ye et al, ${ }^{32}$ the CTR was unable to predict the prognosis of lung $\mathrm{ADC}$, and the authors suggest that part-solid lung $\mathrm{ADC}$ should be defined as one special clinical entity regardless of the percentage of solid components. For these reasons, we simply subclassified the nodules into pure GGNs, PSNs, and SNs. In our study, a solid nodule appearance was revealed to be an independent predictor for STAS. Similar results were reported by other researchers. Shiono et $\mathrm{al}^{13}$ first reported in their study that solid nodules on CT were significantly correlated with STAS $(p=0.032)$. Furthermore, Toyokawa et al $^{17}$ conducted a study to analyze the detailed CT features of patients with lung ADC. They showed that the presence of notches (OR, 1.93; $p=0.01)$ and the absence of GGOs (OR, $0.37 ; p<0.01)$ were independently associated with the presence of STAS. To date, as a radiologist, Kim et $\mathrm{al}^{21}$ conducted the first propensity-matched study for predicting STAS in lung ADC through CT imaging. They revealed that, apart from the solid component, STAS was also significantly associated with the CT features of central low attenuation, illdefined opacity, and air bronchogram. The researchers also found that the percentage of solid components was an independent predictor of STAS (OR, 1.06; 95\% CI, 1.03-1.08) with a sensitivity of $89.2 \%$ and a specificity of $60.3 \%$. Notably, in the study conducted by Kim et al, no STAS was found in the pure GGN cases, which is similar to our results. The rare incidence of STAS in pure GGNs highlights the minimally invasive features of GGNs.

Given that the pathological diagnosis of SMPs and radiological features of SNs were both significant predictors for the presence of STAS in lung ADC and the inconvenience and relative immature method of frozen section pathology to diagnose STAS, we then conducted a combined analysis of the presence or absence of SNs with SMPs and obtained an OR of 10.922 (95\% CI, 5.826-20.475) for STAS in SN (+)/SMP (+) lung ADCs. This indicates that tumors with a pure solid appearance and SMP were approximately ten times more likely to present with STAS than tumors without either an SN or SMP. Surgeons should be aware that for SNs with SMPs according to intraoperative frozen section pathology, limited resection should be cautiously selected regardless of the tumor size due to the high probability for STAS.

To the best of our knowledge, this is the first comprehensive study to evaluate STAS with clinicopathological and radiological features of lung $\mathrm{ADC}$ using propensity score matching. However, several limitations still exist in this study. First, given the nature of this nonrandomized and retrospective study, certain biases may still exist even though we performed propensity score matching. Second, we enrolled ADC patients and excluded only those with squamous or small cell lung cancer, which were recently reported to be associated with the presence of STAS as well. Third, as an important feature with the strongest correlation with pathological pattern or prognosis, the solid appearance of nodules was mainly analyzed, however, further analysis was lack in the percentage of solid components in PSNs as well as other radiological features, such as air bronchograms, spiculation or calcifications. Recent studies ${ }^{33,34}$ regarding using radiomics to assess lung nodules (especially the STAS) have reported inspiring results and may offer guide for our following research. Moreover, due to the limited follow-up period, we failed to analyze the prognostic effect and related factors in this study, which warrant further evaluation. To gain a comprehensive understanding of STAS, well-designed prospective studies are needed to increase the number of cases and broaden the histological subtypes.

In conclusion, we revealed that STAS is a certain pathological entity that correlates with several invasive clinicopathological parameters, especially the pathological diagnosis of SMPs and radiological features of SNs, which may help predict the presence of STAS. 


\section{Ethical Approval and Statement}

The Cancer Institute and Hospital of the Chinese Academy of Medical Sciences Ethics Committee for Clinical Investigation approved this study and waived the requirement of the patient's informed consent for the retrospective nature of the study. This study was conducted in accordance with the Declaration of Helsinki and the patients' data was anonymized and maintained with confidentiality.

\section{Acknowledgments}

We thank all the patients who contributed to this research. This work was supported by the CAMS Innovation Fund for Medical Sciences (2016-I2M-1-001, 2017-I2M-1-005).

\section{Disclosure}

The authors declare no conflicts of interest for this work.

\section{References}

1. Bray F, Ferlay J, Soerjomataram I, et al. Global cancer statistics 2018: GLOBOCAN estimates of incidence and mortality worldwide for 36 cancers in 185 countries. CA Cancer J Clin. 2018;68:394-424. doi: $10.3322 /$ caac. 21492

2. Warth A, Muley T, Kossakowski CA, et al. Prognostic impact of intra-alveolar tumor spread in pulmonary adenocarcinoma. Am J Surg Pathol. 2015;39:793-801. doi:10.1097/PAS.0000000000000409

3. Travis WD, Brambilla E, Nicholson AG, et al. The 2015 World Health Organization classification of lung tumors. $J$ Thorac Oncol. 2015;10:1243-1260. doi:10.1097/JTO.0000000000000630

4. Kadota K, Nitadori JI, Sima CS, et al. Tumor spread through air spaces is an important pattern of invasion and impacts the frequency and location of recurrences after limited resection for small stage I lung adenocarcinomas. $J$ Thorac Oncol. 2015;10:806-814. doi:10.1097/JTO.0000000000000486

5. Masai K, Sakurai H, Sukeda A, et al. Prognostic impact of margin distance and tumor spread through air spaces in limited resection for primary lung cancer. $J$ Thorac Oncol. 2017;12:1788-1797. doi:10.1016/j.jtho.2017.08.015

6. Lee JS, Kim EK, Kim M, et al. Genetic and clinicopathologic characteristics of lung adenocarcinoma with tumor spread through air spaces. Lung Cancer. 2018;123:121-126. doi:10.1016/j. lungcan.2018.07.020

7. Hans B, Russell PA, Jones KD, et al. Pulmonary loose tumor tissue fragments and spread through air spaces (STAS): invasive pattern or artifact? A critical review. Lung Cancer. 2018;123:107-111. doi:10.1016/j.lungcan.2018.07.017

8. Macmahon H, Naidich DP, Goo JM, et al. Guidelines for management of incidental pulmonary nodules detected on CT images: from the Fleischner Society 2017. Radiology. 2017;284:228-243. doi:10.1148/radiol.2017161659

9. Hu SY, Hsieh MS, Hsu HH, et al. Correlation of tumor spread through air spaces and clinicopathological characteristics in surgically resected lung adenocarcinomas. Lung Cancer. 2018;126:189-193. doi:10.1016/j.lungcan.2018.11.003

10. Rami-Porta R, Asamura H, Travis WD, Rusch VW, editors. American joint committee on cancer, lung. In: AJCC Cancer Staging Manual. 8th. New York: Springer; 2017:431-456.
11. Austin PC. Balance diagnostics for comparing the distribution of baseline covariates between treatment groups in propensity-score matched samples. Stat Med. 2009;28:3083-3107. doi:10.1002/sim.3697

12. Onozato ML, Kovach AE, Yeap BY, et al. Tumor islands in resected early-stage lung adenocarcinomas are associated with unique clinicopathologic and molecular characteristics and worse prognosis. $\mathrm{Am}$ J Surg Pathol. 2013;37:287-294. doi:10.1097/PAS.0b013e31826885fb

13. Shiono S, Yanagawa N. Spread through air spaces is a predictive factor of recurrence and a prognostic factor in stage I lung adenocarcinoma. Interact Cardiovasc Thorac Surg. 2016;23:567-572. doi:10.1093/icvts/ivw211

14. Dai C, Xie H, Su H, et al. Tumor spread through air spaces affects the recurrence and overall survival in patients with lung adenocarcinoma $>2$ to $3 \mathrm{~cm}$. $J$ Thorac Oncol. 2017;12:1052-1060. doi:10.1016/j. jtho.2017.03.020

15. Uruga H, Fujii T, Fujimori S, et al. Semiquantitative assessment of tumor spread through air spaces (STAS) in early-stage lung adenocarcinomas. $J$ Thorac Oncol. 2017;12:1046-1051. doi:10.1016/j.jtho.2017.03.019

16. Toyokawa G, Yamada Y, Tagawa T, et al. Significance of spread through air spaces in resected pathological stage I lung adenocarcinoma. Ann Thorac Surg. 2018;105:1655-1663. doi:10.1016/j.athoracsur.2018.01.037

17. Toyokawa G, Yamada Y, Tagawa T, et al. Computed tomography features of resected lung adenocarcinomas with spread through air spaces. $J$ Thorac Cardiovasc Surg. 2018;156:1670-1676. doi:10.1016/j.jtcvs.2018.04.126

18. Maeshima AM, Tochigi N, Yoshida A, et al. Histological scoring for small lung adenocarcinomas $2 \mathrm{~cm}$ or less in diameter: a reliable prognostic indicator. $J$ Thorac Oncol. 2010;5:333-339. doi:10.1097/ JTO.0b013e3181c8cb95

19. Yanagawa N, Shiono S, Abiko M, et al. The clinical impact of solid and micropapillary patterns in resected lung adenocarcinoma. J Thorac Oncol. 2016;11:1976-1983. doi:10.1016/j.jtho.2016.06.014

20. Qian F, Yang W, Wang R, et al. Prognostic significance and adjuvant chemotherapy survival benefits of solid or micropapillary pattern in resected stage IB lung adenocarcinoma patients. $J$ Thorac Cardiovasc Surg. 2017;155:1227-1235. doi:10.1016/j.jtcvs.2017.09.143

21. Kim SK, Kim TJ, Chung MJ, et al. Lung adenocarcinoma: CT features associated with spread through air spaces. Radiology. 2018;289:831-840. doi:10.1148/radiol.2018180431

22. Morimoto J, Nakajima T, Suzuki H, et al. Impact of free tumor clusters on prognosis after resection of pulmonary adenocarcinoma. Thorac Cardiovasc Surg. 2016;152:64-72. doi:10.1016/j.jtcvs.2016.03.088

23. Shiono S, Endo M, Suzuki K, et al. Spread through air spaces is a prognostic factor in sublobar resection of non-small cell lung cancer. Ann Thorac Surg. 2018;106:354-360. doi:10.1016/j. athoracsur.2018.02.076

24. Bains S, Eguchi T, Warth A, et al. Procedure-specific risk prediction for recurrence in patients undergoing lobectomy or sublobar resection for small $(\leq 2 \mathrm{~cm})$ lung adenocarcinoma: an international cohort analysis. J Thorac Oncol. 2019;14:72-86. doi:10.1016/j.jtho.2018.09.008

25. Kameda K, Lu S, Eguchi T, et al. Can tumor spread through air spaces (STAS) in lung adenocarcinomas be predicted pre- and intraoperatively. J Thorac Oncol. 2017;12:411-412. doi:10.1016/j.jtho.2016.11.473

26. Walts AE, Marchevsky AM. Current evidence does not warrant frozen section evaluation for the presence of tumor spread through alveolar spaces. Arch Pathol Lab Med. 2018;142:59-63. doi:10.5858/arpa.20160635-OA

27. Eguchi T, Kameda K, Lu S, et al. Lobectomy is associated with better outcomes than sublobar resection in spread through air spaces (STAS)-positive T1 lung adenocarcinoma: a propensity score-matched analysis. J Thorac Oncol. 2019;14:87-98. doi:10.1016/j.jtho.2018.09.005

28. Cho JH, Choi YS, Kim J, et al. Long-term outcomes of wedge resection for pulmonary ground-glass opacity nodules. Ann Thorac Surg. 2015;99:218-222. doi:10.1016/j.athoracsur.2014.07.068 
29. Hattori A, Matsunaga T, Takamochi K, et al. Importance of ground glass opacity component in clinical stage IA radiologic invasive lung cancer. Ann Thorac Surg. 2017;104:313-320. doi:10.1016/j. athoracsur.2017.01.076

30. Matsunaga T, Suzuki K, Takamochi K, et al. What is the radiological definition of part-solid tumour in lung cancer. Eur $J$ Cardiothorac Surg. 2017;51:242-247. doi:10.1093/ejcts/ezw344

31. Yip R, Li K, Liu L, et al. Controversies on lung cancers manifesting as part-solid nodules. Eur Radiol. 2018;28:747-759. doi:10.1007/ s00330-017-4975-9
32. Ye T, Deng L, Wang S, et al. Lung adenocarcinomas manifesting as radiological part-solid nodules define a special clinical subtype. J Thorac Oncol. 2019;14:617-627. doi:10.1016/j.jtho.2018.12.030

33. Chen D, She Y, Wang T, et al. Radiomics-based prediction for tumor spread through air spaces in stage I lung adenocarcinoma using machine learning. Eur J Cardiothorac Surg. 2020;58:51-58. doi:10.1093/ejcts/ezaa011

34. Jiang C, Luo Y, Yuan J, et al. CT-based radiomics and machine learning to predict spread through air space in lung adenocarcinoma. Eur Radiol. 2020;30:4050-4057. doi:10.1007/ s00330-020-06694-z

\section{Publish your work in this journal}

Cancer Management and Research is an international, peer-reviewed open access journal focusing on cancer research and the optimal use of preventative and integrated treatment interventions to achieve improved outcomes, enhanced survival and quality of life for the cancer patient.
The manuscript management system is completely online and includes a very quick and fair peer-review system, which is all easy to use. Visit http://www.dovepress.com/testimonials.php to read real quotes from published authors. 